\title{
A model of auditory deviance detection
}

\author{
Emine Merve Kaya and Mounya Elhilali \\ Department of Electrical and Computer Engineering \\ The Johns Hopkins University \\ Baltimore, MD 21218, USA \\ Email: \{merve, mounya\}@jhu.edu
}

\begin{abstract}
A key component in computational analysis of the auditory environment is the detection of novel sounds in the scene. Deviance detection aids in the segmentation of auditory objects and is also the basis of bottom-up auditory saliency, which is crucial in directing attention to relevant events. There is growing evidence that deviance detection is executed in the brain through mapping of the temporal regularities in the acoustic scene. The violation of these regularities is reflected as mismatch negativity (MMN), a signature electrical response observed using electro-encephalograpy (EEG) or magneto-encephalograpy (MEG). While numerous experimental results have quantified the properties of this MMN response, there have been few attempts at developing general computational frameworks of MMN that can be integrated in comprehensive models of scene analysis. In this work, we interpret the underlying mechanism of the MMN response as a Kalman-filter formulation that provides a recursive prediction of sound features based on the past sensory information; eliciting an MMN when predictions are violated. The model operates in a high-dimensional space, mimicking the rich set of features that underlie sound encoding up the level of auditory cortex. We test the proposed scheme on a variety of simple oddball paradigms adapted to various features of sounds: Pitch, intensity, direction, and inter-stimulus interval. Our model successfully finds the deviant onset times when the deviant varies from the standard in one or more of the calculated dimensions. Our results not only lay a foundation for modeling more complex elicitations of MMN, but also provide a versatile and robust mechanism for outlier detection in temporal signals and ultimately parsing of auditory scenes.
\end{abstract}

\section{INTRODUCTION}

As we hear our surrounding acoustic scene, the auditory system is continuously tracking the events in the scene and classifying them as standards and deviants based on a notion of regularity. There is evidence that this information extraction occurs regardless of our paying attention to the sounds around us. In electro-encephalograpy (EEG) and magneto-encephalograpy (MEG) recordings of the brain, the determinant of regularity appears as a negative difference in the N1 responses between the standard and deviant sound. This difference is called the mismatch negativity (MMN) and has been studied since its discovery over 30 years ago [1]. However, while there is an extensive literature on what type of stimuli elicit the MMN response, the mechanisms underlying it are still only theories.

MMN can be interpreted as a physiological index of auditory saliency. It has been shown that MMN can be observed in asleep and comatose states, suggesting that it is a preattentive response. [2] [3] This coincides with the notion of bottom-up saliency, which is assumed to occur prior to topdown influences. [4] [5] Additionally, although MMN has been primarily studied in the auditory system, there is evidence for a visual counterpart; the modality that is mainly considered for saliency in the brain. [6] The computational uses of an understanding of the underlying mechanisms of MMN become apparent with this drawn parallel.

From a computational perspective, we can view the mechanism of MMN extraction as a outlier detection scheme. Given the input sound signal, the system is able to find which sound events do not fit in with the perceived standard in the signal. This is in fact what is physiologically extracted in the brain and the time instance of the deviant sound is reflected in the elicitation of the MMN; the same type of information that is being captured in previous auditory saliency maps in the literature. [7] The knowledge of this process as it occurs in the brain would lead us to build efficient models for deviance detection that would fit in greater scene analysis models. [8] [9] [10] Since this information is yet unknown, we will build our computational model based on one theory of MMN elicitation that, while it does not provide a low-level explanation of the processes in the brain, has common points with the overall frameworks of the most prominent theories in the literature. In this work, we are not presenting a new theory of $\mathrm{MMN}$, but rather a concrete computational mechanism that implements the selected scheme.

There are three theoretical models of MMN proposed in the literature that we will consider. First, the original assumption of MMN elicitation, which is based on a memory trace in the brain. [11] The representations of incoming stimuli as they emerge in the brain are stored in a sensory memory trace. New representations as they appear are compared to the memory, and an MMN is elicited if there is a mismatch. The second theory of MMN, which was proposed concurrently as the first but had been pushed aside until recent experimental evidence, is the adaptation model. [12] According to this model, MMN can be explained as simple as neuronal adaptation that occurs already in the brain and no separate mechanism is necessary. It is characteristic of neuronal responses to be attenuated as they are constantly stimulated. Thus the standard in the scene will have a suppressed response. The deviant in the scene, due to its nature of being different from the standard, will activate a separate set of neurons, which will result in an increased neuronal response, and this sudden difference is referred to as MMN. The third, more recent, theory ("predictive coding") 


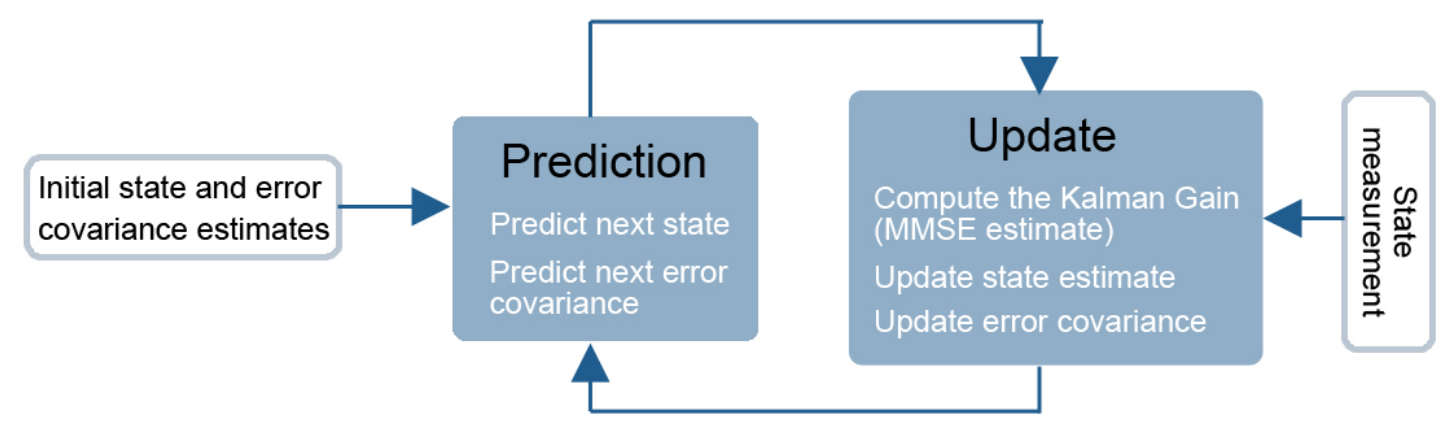

Fig. 1: The advance of a Kalman filter. First the initial state and error covariance are estimated from a buffer of samples. Using these, the next state and error covariance are predicted according to the Kalman model. This prediction is updated with the incoming real measurement of the state. The update is such that it minimizes the mean-square error between the prediction and measurement. These updates are once again used to predict the next state, and the filter iterates the same way with each time increment.

can be seen as a comprehensive explanation of MMN considering the range of stimuli that elicit MMN that the previous models have a difficult time explaining. The predictive coding model assumes that as we hear the sound, some notion of regularity is extracted in the brain, which adapts over time to the received input. The MMN is elicited when a sound occurs violates this notion of regularity. [13] This scheme is supported by the general notion that predictive coding is a submechanism of perception in the brain. [14] The model we implement here is based on this framework. Our model is merely an alternative interpretation of this framework with the goal of integration into larger computational models. Different interpretations of this framework for various applications can be found in the literature. [15] [16]

\section{THE KALMAN-MMN MODEL}

We base our implementation on the Kalman filter due to it being straightforward to implement and interpret, fast and efficient, requiring little memory, and robust to noisy realworld data. The Kalman filter is the basic block that realizes the notion of predictive coding. It is a discrete-time linear dynamical model on a Markov chain; with added Gaussian noise. The filter is a recursive advance that predicts the value of a state, along with error covariance (measure of the accuracy of the estimate), and refines the estimate based on measurements of the state. This is essentially what the predictive coding scheme is and it should be evident that the Kalman filter provides a direct implementation of the framework. The possibility of a Kalman filter type of mechanism existing in the brain has been previously demonstrated. [17] [18]

The underlying process of a Kalman model is given in 1 . $X$ is the state that is being tracked. This may be understood as a feature of sound that is tracked in the brain, eliciting the MMN response when a deviance is detected. $Y$ is the real measurement of this state $X$. The recursive Markov nature of the filter is demonstrated by the state at time $t$ depending solely on the state at time $t-1$. The measurement is inherently a function of the real state value. Both the state and measurement are perturbed by noise, respectively $w$ and v. $F$ and $H$ represent time-dependent system matrices that tie the elements of the model together. The Kalman filter advance is demonstrated in Fig.1.

$$
\begin{aligned}
X_{t} & =F_{t-1} X_{t-1}+w_{t-1} \\
Y_{t} & =H_{t} X_{t}+v_{t}
\end{aligned}
$$

Let us assume that we already have a feature dimension that we are working on, such as time of onset. Our stimulus may be an oddball paradigm with a regularly repeating tone and a deviant tone that is delayed. The time of onset $A_{n}$ for each tone can be calculated recursively as a function of the time between consecutive tones $B_{n}$. The step increment in this setup represents the count of tones. The system described can be modelled as in 2, where $w_{n}$ takes care of small jitter in the regularity. Our measurement in this model $Y_{n}$ is simply the time of onset, complemented by a small measurement noise. We model all of the noise variables as Gaussian white noise with distinct variances.

$$
\begin{aligned}
A_{n} & =A_{n-1}+B_{n-1}+w_{n-1} \\
Y_{n} & =A_{n}+v_{n}
\end{aligned}
$$

Given these equations we can directly fit them into the Kalman model framework with the adjustments given in 3 .

$$
X_{n}=\left[\begin{array}{l}
A_{n} \\
B_{n}
\end{array}\right], F_{n}=\left[\begin{array}{ll}
1 & 1 \\
0 & 1
\end{array}\right], H_{n}=\left[\begin{array}{ll}
1 & 0
\end{array}\right]
$$

The system matrices are constant in our case. Given $Q$, the system noise covariance and $R$, measurement covariance, we can derive the Kalman gain for this system that gives the MMSE estimate, which we denote $K_{n} . \hat{P}_{n}$ is the state prediction error covariance matrix. $\hat{X}_{n}$ represents the a posteriori estimate of the state $X_{n}$. Putting these all together, we have the final recursive system that will be hereafter referred only as the "Kalman", presented in 4. Intermediate values such as 
a priori state estimate and innovation have been incorporated into the equations for conciseness.

$$
\begin{aligned}
K_{n} & =\left(F \hat{P} F^{T}+Q\right) H^{T}\left(H\left(F \hat{P} F^{T}+Q\right) H^{T}+R\right)^{-1} \\
\hat{X}_{n} & =F_{n-1} \hat{X}_{n-1}+K_{n}\left(Y_{n}-H F \hat{X}_{n-1}\right. \\
\hat{P}_{n} & =\left(I-K_{n} H\right)\left(F \hat{P}_{n-1} F^{T}+Q\right)
\end{aligned}
$$

Even though we derived this model with one specific feature in mind, the same set of equations adapt seamlessly to any feature. We will simply denote $B_{n}$ to be the value of the feature at some time instance. For features other than time onset, we can have the step $n$ be real time increments. The initial values of the system are calculated from the first few samples of the feature signal. The variances of the noise parameters are tuned to match the results from the literature, shown in Section III.

Now that we have our building block for the model, we can use it to implement a system that will output MMN for the various conditions presented in the literature. [19] The Kalman-MMN model begins with putting the input sound signal through feature detection that extracts features such as frequency, intensity, duration, location, etc. The prediction system will work separately on each one of these features. Additionally, one tracker will work on all of the features simultaneously to detect complex invariances. In this work, we will not concern ourselves with the extraction of these features, but assume that we have a set of numbers for the extracted feature. Our goal is to demonstrate that given that the features are extracted, we will be able to produce the MMN response. The working of the tracking system will be outlined below.

First, the standard of the incoming sound feature is detected within an initial buffer. For each detected stream, two kalman trackers are initialized: One tracks the incoming feature value, one tracks the timing of the values. So, every "value" Kalman will be predicting only one value, and every "time" Kalman will be predicting the onset times of only one value in the feature stream.

At each time, the incoming value is compared with all of the predictions. If no tracking filter has predicted this value, MMN occurs, and a new stream is initialized so that the value may form a new stream if it occurs again not long after. If the value was predicted by a Kalman, it will also be compared against the "time" Kalman for that stream. MMN is elicited if the time occurrence is far from the prediction of the "time" Kalman. The decision of whether an input value fits into the prediction of a Kalman depends on the measurement innovation covariance, a measure of how well the predictions are matching the input. This is a direct function of the estimated error covariance, which is updated at every time step. The result of this is that if the innovation is always small, the tolerance for fitting a Kalman will get smaller over time. But if the input signal is very noisy, the tolerance will grow.

Finally, a memory cleanup exists to remove the tracking of
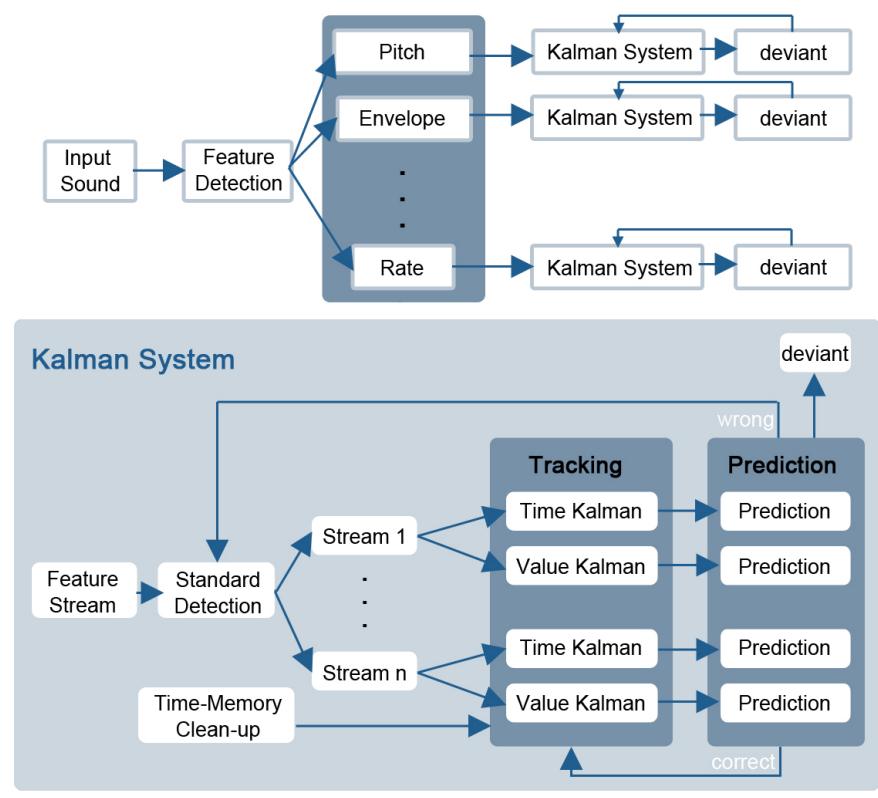

Fig. 2: The full Kalman-MMN model.

the streams that have not been updated for a long time. This entire system is summarized in Fig.2.

\section{RESULTS}

We demonstrate the results of the model on some selected experimental stimuli in the literature that have shown the elicitation of MMN for their stimuli. We will follow the outline presented in [19] that groups the data in the literature into classes of stimuli that elicit MMN. All stimuli are of regularly repeating tones in an oddball paradigm. The classes are as follows. Simple invariance occurs when the standards are the same in all features, and the deviant is different in one feature. This can be likened to a sequence of letters such as $A A A A B$. Pattern invariance is when there is a pattern of tones regularly repeating instead of a single tone, and the deviance is the breaking of the pattern, such as $A B A B A B A B B A$. Hypercomplex invariance has the standard tones having a few possible combinations of values among features, and the deviant has a combination previously unseen, such as $(A, C)(A, C)(B, D)(B, D)(A, C)(A, D)$. Complex invariance is the existence of simple invariance among one feature, and the lack of any standard on other features. The deviant will be on the feature that the simple invariance was on, such as $(A, A)(B, A)(C, A)(D, A)(D, A)(B, C)$. The last class considered is abstract invariance, which is concerned with relationships between tones rather than the actual feature of the tones. An example would be that pairs of stimuli are presented where the second tone has a higher pitch than the first. The deviant would have a decreasing pitch rather than increasing. We will not cover this type of invariance here because it cannot be summarized with just one example, however, we can still model this type of invariance with the Kalman-MMN model if we are able to determine the relationship between stimuli. In the previous example, we can 


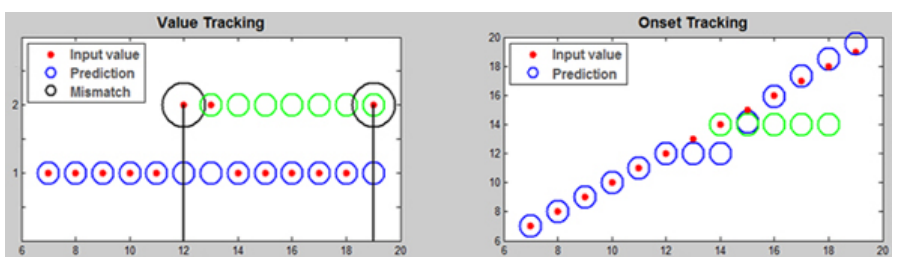

(a) Simple invariance sequence 1..1221..12. The input value is either 1 or 2 . This is the values among time of an abstract feature. MMN is elicited from the value tracking Kalman.

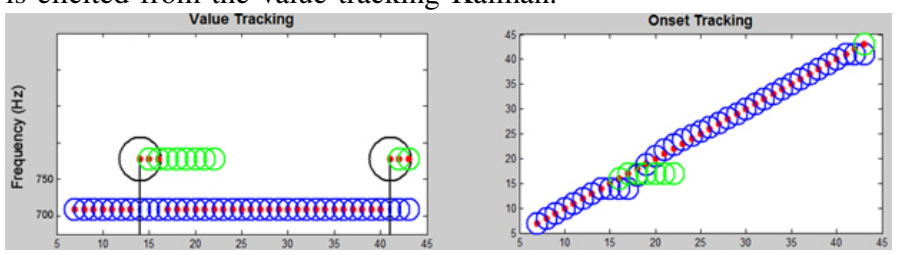

(c) Simple invariance stimuli used in [20]. The standard stimulus $(\mathrm{P}=0.9)$ was a $700 \mathrm{~Hz}$ tone. Block of ISI $350 \mathrm{~ms}$ and intensity $55 \mathrm{~dB}$ chosen. The deviant stimulus $(\mathrm{P}=0.1)$ only differed in frequency by $50 \mathrm{~Hz}$

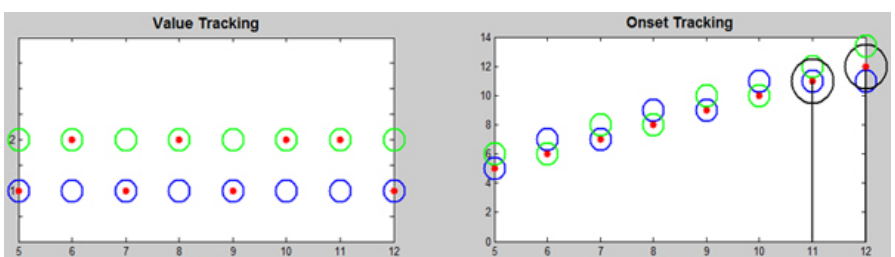

(b) Pattern invariance sequence $12 \ldots 12121221$. The input value is either 1 or 2 . This is the values among time of an abstract feature. MMN is elicited from the time tracking Kalman.

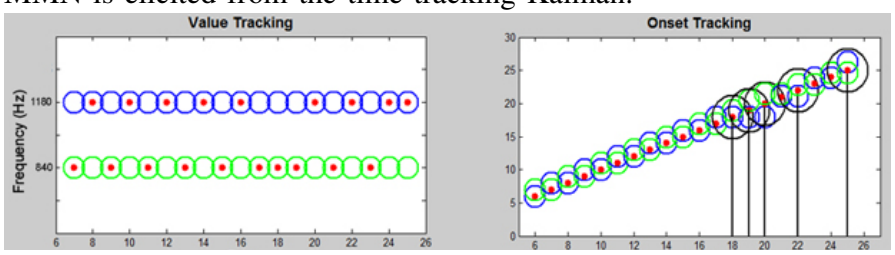

(d) Pattern invariance stimuli used in [21]. Tone sequences consisted of two tones that alternated regularly. Occasionally, one of the stimuli were repeated. In one condition, the two tones were separated by six semitones: $841 \mathrm{~Hz}$ and $1189 \mathrm{~Hz}$.

Fig. 3: Demonstration of the Kalman-MMN model running on sequences where the invariance is in only one feature. The model computes all separate features, along with integration of features, but they are not shown because the Kalman's only track the same value among those dimensions and there is no MMN output. Shown with the red dots is the actual value in the sequence at that time instance. The time starts at 6 because the values before then are used to determine the standard of the sequence, and the prediction begins at time point 6 (the buffer size is arbitrary). The colored circles are the predicted values by a Kalman, each color representing a different Kalman. The black circles are the time instances where the model finds a mismatch.

simply track the pitch difference between stimuli rather than the pitch values. With this adjustment, it turns into a simple invariance problem.

Let us explain how each of these cases work out with the Kalman-MMN model. First, we code the simple invariance as a series of tones that are the same on an abstract dimension, with a value of 1 . The deviant will have the value 2 . This is the same as the character string example, except we have now replaced the letters with numbers so we can mathematically track them. The first few iterations of the Kalman-MMN model are shown in Fig.3(a). At time 12, when the value changes from 1 to 2 , there is only one Kalman running, tracking value 1 and it correctly recognizes that there is a mismatch. Following this, a new Kalman is created tracking value 2. It stops tracking value 2 when it does not reoccur for a while, so when we get the third 2, it elicits an MMN again. It should be noted that the size of this tracking memory is not constant throughout all examples and can be modified as necessary. Estimating what this value should be is beyond the scope of this work.

In Fig.3(c) we have the same paradigm but now it is working on real feature values as used in the referenced study rather than the example values of 1 and 2 . The result is the same as the example run of the model.

The next case is pattern invariance. The run of the model for the abstract case is shown in Fig.3(b). There is no MMN elicitation at the value Kalmans (one Kalman tracking the value 1, one Kalman tracking the value 2) but there is on both time Kalmans. This is because due to the breaking of the pattern, the onset times of both of the unique values have been disrupted from their regularity, so each time Kalman associated with one of the values gives an MMN elicitation. A side point demonstrated in this example is that, due to our determining of the standard before the estimation begins, we are already expecting both 1 and 2 as possible values and the estimation starts off with two value Kalmans rather than one. Contrast this with the simple invariance case where we expected only one value at the beginning of estimation.

Fig.3(d) the same paradigm is shown on real feature values as used in the referenced study rather than the example values of 1 and 2. The result is again the same as the example run of the model, but noisier due to the more complicated nature of the real stimuli vs. the abstract stimuli.

The following cases take multiple features into consideration when defining the standard and deviant. The results of the model for hypercomplex invariance for the abstract case is shown in Fig.4(a). The first two rows of the value Kalmans have no MMN elicitations because all of the values shown are possible expected values among their separate dimensions. In the feature integration Kalman, however, we see the MMN output. In this dimension, the value being tracked is multidimensional. One Kalman is tracking $(2,3)$ and one Kalman is tracking $(1,2)$. The read value at time 13 fits neither, so it is recorded as an outlier.

The results for this case on real values are shown in Fig.4(c). Again the tracking is noisier due to the nature of the data, but the MMN output at correct places are also apparent here. 
The last case is complex invariance. It should be evident that for our model complex invariance is almost the same as simple invariance. This is because we extract many features and follow them all at once, no matter where the invariance is. The difference between the two is the assumption in simple invariance that the other features will have the same value in the standard and deviant, whereas in complex invariance, they may be varying with no pattern. This does not computationally make a difference for the Kalman-MMN model. Since the values are greatly varying in the buffer, the standard at the beginning of estimation will have a large expected variance. This means that even though they are different values, they will not be regarded as outliers in the Kalman filter. So again, only the feature that has the standard will elicit an MMN response. This is shown in Fig.4(b) and Fig.4(d). Additionally, note that the estimate value for the no-standard feature is varying over time, adapting to the input. Since the variance of values is retained over time, the tolerance remains high.

\section{Discussion}

In this section we take a look at how our implementation compares to the MMN frameworks described in Section I.

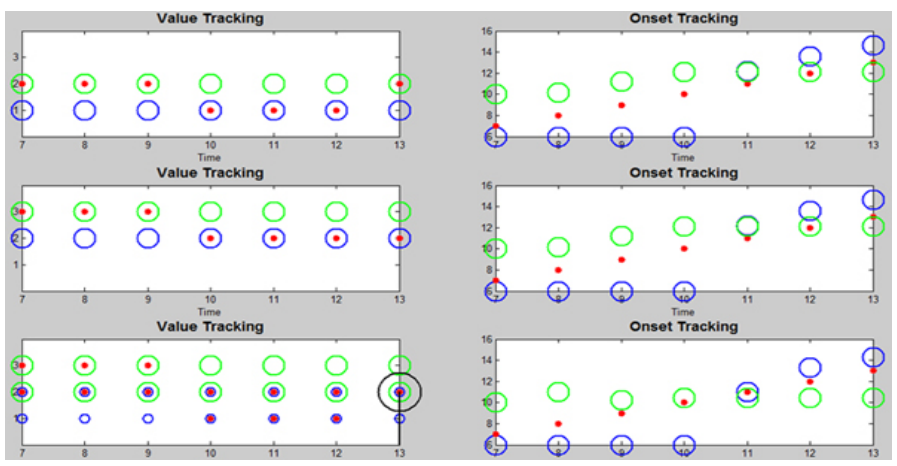

(a) Hypercomplex invariance sequence $(2,3)(2,3)(2,3)(1,2)(1,2)(1,2)$ $(2,2)$. The input value among each abstract feature is 1,2 or 3 . MMN is elicited from the value tracking Kalman on the integration of features.

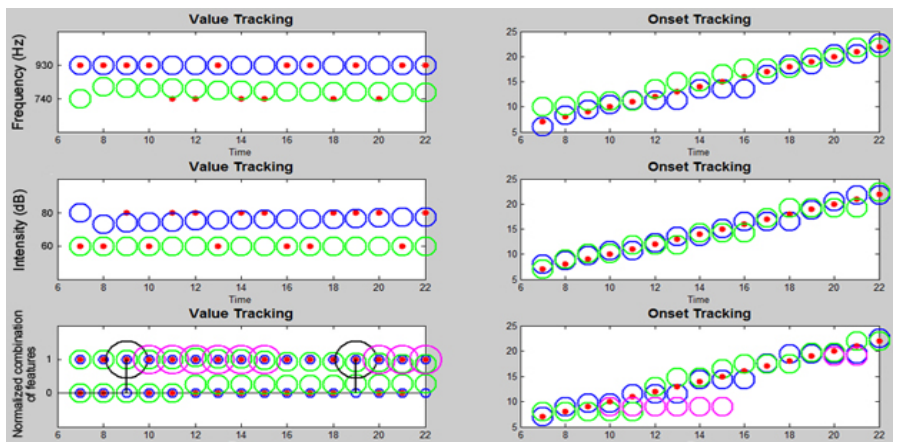

(c) Hypercomplex invariance stimuli used in [22]. Two standard tones $(\mathrm{P}=0.45$ each) were $932 \mathrm{Haz}$ and $80 \mathrm{~dB}$; and $739 \mathrm{~Hz}$ and $60 \mathrm{~dB}$. Deviants have the frequency of one and the intensity of the other ( $\mathrm{P}=0.05$ each).
Although some attributes of our procedure are common to all predictive coding models, here we illustrate the points that are specific to the implementation presented in this work.

Similar to the memory trace model, we detect transient events along with looking for outlier values in the features of the incoming sound. However, the memory trace model holds these incoming values in an "auditory memory" and explicitly compares against memory content. Here, we do not actually save the value that the Kalman system is tracking, it is implicit in the system matrices of the Kalman setup; we merely check whether the incoming value fits the prediction of a tracker.

Although our model is not based on neuronal adaptation explicitly, we may draw similarities on the general procedure to this framework. Just as neurons that are continuously firing for standards adapt over time, our Kalman trackers get better prediction and lower tolerance the more they are updated. A deviant stimulates a new set of neurons that were not previously activated; and in our model, a new Kalman begins responding to the deviant value. In our model, as is also the case in the adaptation model, the concept of $\mathrm{N} 1$ and $\mathrm{MMN}$ is essentially the same.

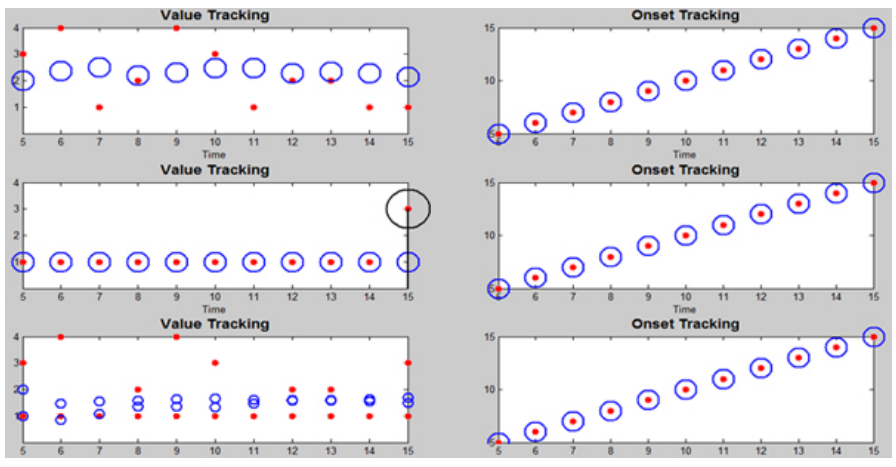

(b) Complex invariance sequence $(3,1)(4,1)(1,1)(2,1)(4,1)(3,1)(1,1)(2$, $1)(2,1)(1,1)((1,2)$. The input value among each abstract feature is 1 , 2,3 or 4 . MMN is elicited from the value tracking Kalman on the second feature.

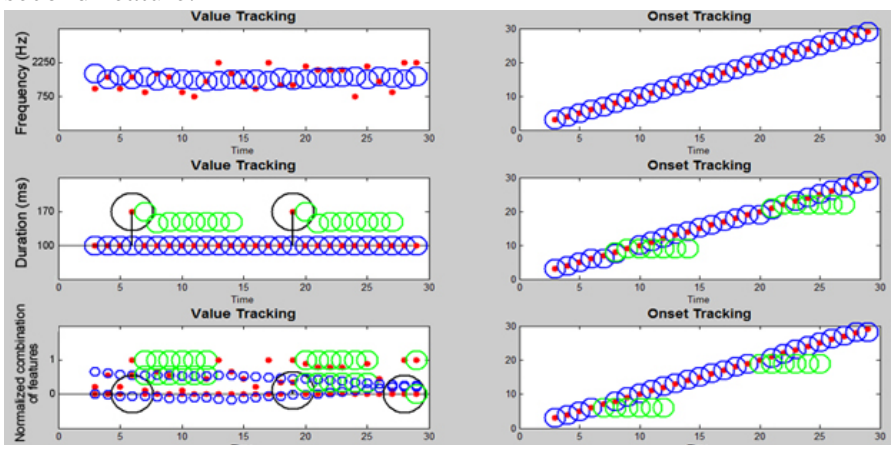

(d) Complex invariance stimuli used in [23]. $90 \%$ of the tones were $100 \mathrm{~ms}$ in duration, $10 \%$ were $170 \mathrm{~ms}$. In one condition the tones varied over a range of tonal frequencies. Thus there was no "standard" tone.

Fig. 4: Demonstration of the Kalman-MMN model running on sequences where the invariance is in multiple features. Only the relevant feature dimensions are shown. In all figures, the top two rows are demonstrations of the separate features, and the last row shows the integration of the presented features. The figure notations are identical to those used in Fig.3. 
From the perspective of general predictive coding schemes, we can see that even though we do not have an explicit hierarchical structure, the two figures in Fig.2 can be seen as lower level (first figure) and higher level (bottom figure - Kalman section) processing. A model employing Bayesian processes has been previously proposed [24] which is more concerned with a physiological explanation, whereas we are concerned with efficient implementation and use in scene analysis frameworks.

A related note can be made about the attention framework in [25], which our model readily fits into. Similarly to that framework, the basis of our prediction (and therefore the "MMN" generation) depends on the standard formation. We can accordingly incorporate effects of attention on our model: Attention can be seen as an external input altering the method of standard formation; after that the proceedings of the model need not change.

\section{CONClusion}

This work presents a model that is biologically motivated, yet versatile and easy enough in implementation to incorporate into other computational models that might require deviance detection in sound. (auditory scene analysis models, computation of saliency, etc.)

Our model can also be seen as an initial step to building a computational model of MMN based on Kalman filters. By tuning the model to match the results of the numerous studies in the MMN literature, we may be able to obtain a system that accurately captures the essence of MMN production. The end goal would be to obtain a system that replicates the experimental results on the given stimulus, which would aid in further elucidating the properties of the MMN response.

This work can be interpreted as providing support for the predictive coding theory of MMN elicitation. We have shown that following the predictive coding framework, we are able to match the results of many experiments in the literature; making up for the shortcomings of both the memory trace model and adaptation model by following a union of the two models.

\section{ACKNOWLEDGMENTS}

This research was supported by NSF grant IIS-0846112, NIH grant 1R01AG036424 and ONR grants N000141010278 and N00014-12-1-0740.

\section{REFERENCES}

[1] R. Näätänen, A. Gaillard, and S. Mäntysalo, "Early selective-attention effect on evoked potential reinterpreted," Acta Psychologica, vol. 42, no. 4 , pp. $313-329,1978$.

[2] M. Sallinen, J. Kaartinen, and H. Lyytinen, "Is the appearance of mismatch negativity during stage 2 sleep related to the elicitation of k-complex?" Electroencephalography and Clinical Neurophysiology, vol. 91, no. 2, pp. 140 - 148, 1994.

[3] N. Kane, S. Curry, S. Butler, and B. Cummins, "Electrophysiological indicator of awakening from coma," The Lancet, vol. 341, no. 8846, pp. $688-, 1993$

[4] L. Itti and C. Koch, "Computational modelling of visual attention," Nat Rev Neurosci, vol. 2, no. 3, pp. 194-203, 032001.

[5] A. K. Engel, P. Fries, and W. Singer, "Dynamic predictions: Oscillations and synchrony in top-down processing." Nature Reviews Neuroscience, vol. 2, no. 10, pp. $704-716,2001$.
[6] P. Pazo-Alvarez, F. Cadaveira, and E. Amenedo, "Mmn in the visual modality: a review," Biological Psychology, vol. 63, no. 3, pp. $199-$ 236, 2003.

[7] E. M. Kaya and M. Elhilali, "A temporal saliency map for modeling auditory attention," in Information Sciences and Systems (CISS), 2012 46th Annual Conference on, march 2012, pp. 1 -6.

[8] R. Näätänen, T. Kujala, and I. Winkler, "Auditory processing that leads to conscious perception: A unique window to central auditory processing opened by the mismatch negativity and related responses," Psychophysiology, vol. 48, no. 1, pp. 4-22, 2011.

[9] I. Winkler, S. L. Denham, and I. Nelken, "Modeling the auditory scene: predictive regularity representations and perceptual objects," Trends in Cognitive Sciences, vol. 13, no. 12, pp. 532 - 540, 2009.

[10] C. Escera and M. J. Corral, "Role of mismatch negativity and novelty-p3 in involuntary auditory attention." Journal of Psychophysiology, vol. 21, no. 3-4, pp. $251-264,2007$.

[11] R. Näätänen, "The role of attention in auditory information processing as revealed by event-related potentials and other brain measures of cognitive function," Behavioral and Brain Sciences, vol. 13, pp. 201233, 51990.

[12] P. J. C. May and H. Tiitinen, "Mismatch negativity (mmn), the devianceelicited auditory deflection, explained," Psychophysiology, vol. 47, no. 1, pp. 66-122, 2010

[13] I. Winkler, "Interpreting the mismatch negativity." Journal of Psychophysiology, vol. 21, no. 3-4, pp. 147 - 163, 2007.

[14] K. Friston, "Learning and inference in the brain," Neural Networks, vol. 16, no. 9, pp. 1325 - 1352, 2003 , ice:title ${ }_{i}$ Neuroinformatics $/$ ce:title ${ }_{i}$.

[15] M. I. Garrido, K. J. Friston, S. J. Kiebel, K. E. Stephan, T. Baldeweg, and J. M. Kilner, "The functional anatomy of the mmn: A dem study of the roving paradigm," NeuroImage, vol. 42, no. 2, pp. 936 - 944, 2008.

[16] T. Bohm, R. Mill, A. Bendixen, I. Winkler, and S. Denham, "Competing predictive regularity representations in an abstract model of auditory stream segregation (chains)," International Journal of Psychophysiology, vol. 85 , no. 3, pp. $317-, 2012$.

[17] G. Szirtes, B. Páczos, and A. Lörincz, "Neural kalman filter," Neurocomputing, vol. 65 - 66, no. 0, pp. 349 - 355, 2005.

[18] R. Linsker, "Neural network learning of optimal kalman prediction and control," CoRR, vol. abs/0805.4247, 2008.

[19] T. Picton, C. Alain, L. Otten, W. Ritter, and A. Achim, "Mismatch negativity: Different water in the same river." Audiol Neurootol, vol. 5, pp. 111-139, 2000.

[20] E. Schröger, "The influence of stimulus intensity and inter-stimulus interval on the detection of pitch and loudness changes," Electroencephalography and Clinical Neurophysiology/Evoked Potentials Section, vol. 100 , no. 6, pp. $517-526,1996$.

[21] C. Alain, D. L. Woods, and K. H. Ogawa, "Brain indices of automatic pattern processing," Neuroreport, vol. 6, pp. 140-144, 1994.

[22] R. Takegata, P. Paavilainen, R. Näätänen, and I. Winkler, "Independent processing of changes in auditory single features and feature conjunctions in humans as indexed by the mismatch negativity," Neuroscience Letters, vol. 266, no. 2, pp. 109 - 112, 1999.

[23] H. Gomes, W. Ritter, and H. G. Vaughan, "The nature of preattentive storage in the auditory system," J. Cognitive Neuroscience, vol. 7, no. 1, pp. 81-94, Jan. 1995. [Online]. Available: http://dx.doi.org/10.1162/jocn.1995.7.1.81

[24] M. I. Garrido, J. M. Kilner, K. E. Stephan, and K. J. Friston, "The mismatch negativity: A review of underlying mechanisms," Clinical Neurophysiology, vol. 120, no. 3, pp. 453 - 463, 2009.

[25] E. S. Sussman, "A new view on the mmn and attention debate," Journal of Psychophysiology, vol. 21, no. 3-4, pp. 164-475, 2007. 